\title{
Detection of High-Grade Pancreatic Intraepithelial Neoplasia without Morphological Changes of the Main Pancreatic Duct over a Long Period: Importance for Close Follow-Up for Confirmation
}

\author{
Kentaro Yamao ${ }^{a}$ Mamoru Takenaka $^{a}$ Atsushi Nakai $^{a}$ Shunske Omoto ${ }^{a}$ \\ Ken Kamata ${ }^{a}$ Kosuke Minaga $^{a}$ Takeshi Miyata $^{a}$ Hajime Imai ${ }^{a}$ \\ Toshiharu Sakurai $^{\mathrm{a}}$ Tomohiro Watanabe $^{\mathrm{a}}$ Naoshi Nishida $^{\mathrm{a}} \quad$ Ippei Matsumoto $^{\mathrm{b}}$ \\ Yosihumi Takeyamab ${ }^{b}$ Takaaki Chikugo $^{c}$ Masatoshi Kudo $^{a}$

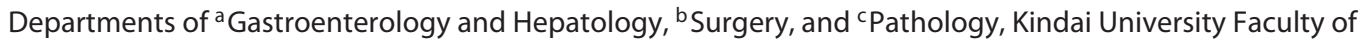 \\ Medicine, Osaka-Sayama, Japan
}

\section{Keywords}

Pancreatic cancer $\cdot$ High-grade pancreatic intraepithelial neoplasia - Carcinoma in situ - Localized main pancreatic duct stenosis · Distal main pancreatic duct dilation

\begin{abstract}
Pancreatic intraepithelial neoplasia (PanIN) is a microscopic papillary noninvasive lesion arising from the pancreatic ductal epithelium. However, the natural history and time to progression of high-grade PanIN remain unclear. Herein, we report 2 cases of high-grade PanIN without morphological changes of the main pancreatic duct (MPD) over relatively long periods. In the first case, a 63-year-old man was identified with MPD dilation. Magnetic resonance cholangiopancreatography showed localized stenosis in the pancreatic body with distal MPD dilation. Endoscopic retrograde pancreatography (ERP) was attempted because of possible highgrade PanIN but was unsuccessful. At 15-month follow-up, there was no change in the form of the MPD in various im-
\end{abstract}

\section{KARGER}

(C) 2017 S. Karger AG, Basel

E-Mail karger@karger.com

www.karger.com/ocl ages. However, ERP was re-performed because of possible high-grade PanIN, and cytology showed adenocarcinoma. Postoperative pathology indicated diffuse lesions corresponding to high-grade PanINs in the MPD stenosis and surrounding branches. Final diagnosis was high-grade PanIN. In the second case, a 77-year-old man was identified with MPD dilation. Magnetic resonance cholangiography showed localized stenosis in the MPD of the pancreatic head with distal MPD dilation. He was diagnosed with MPD stenosis caused by chronic pancreatitis, and further examination was not recommended. At 25 months, the patient was referred to our hospital because of a mild change in MPD dilation. ERP showed localized irregular stenosis in the MPD, and cytology showed suspected adenocarcinoma. Postoperative pathology indicated a localized lesion with high-grade PanIN in the branch duct around the MPD stenosis. Final diagnosis was high-grade PanIN. In conclusion, we report 2 cases of highgrade PanIN without morphological changes of the MPD over relatively long periods. Even if a definite diagnosis is not obtained at initial examination, a strict follow-up observa-

Dr. Mamoru Takenaka

Department of Gastroenterology and Hepatology Kindai University Faculty of Medicine

377-2 Ohno-Higashi, Osaka-Sayama, Osaka 589-8511 (Japan)

E-Mail mamoxyo45@gmail.com 
tional study should be performed. Re-examination, including ERP, should also be considered in cases with risk factors of pancreatic cancer, even if there is no change in MPD form.

(c) 2017 S. Karger AG, Basel

\section{Introduction}

Pancreatic cancer has a poor prognosis, and early diagnosis is important for improved outcomes. Small pancreatic cancers have a better prognosis [1], although detection and diagnosis of noninvasive or small invasive lesions are often challenging. Pancreatic intraepithelial neoplasia (PanIN) is considered a precursor for invasive pancreatic cancer [2]. PanIN is defined as a microscopic papillary or flat and noninvasive epithelial neoplasm arising from the pancreatic ductal epithelium. PanIN is divided into 2 subtypes: low-grade PanIN (previously reported as PanIN-1 and -2) and high-grade PanIN (previously reported as PanIN-3) involving a carcinoma in situ $[2,3]$. As high-grade PanIN does not involve a massforming lesion, its diagnosis is challenging by imaging. Detection of high-grade PanIN involves the presence of an abnormal main pancreatic duct (MPD) form, including localized MPD stenosis with distal MPD dilation, focal ductal branch dilation, and cyst formation. Exacerbation of these findings with tumor progression is also a sign of high-grade PanIN. However, the natural history and time to progression of high-grade PanIN remain unclear. Herein, we report 2 cases of high-grade PanIN without morphological changes in the MPD over relatively long periods.

\section{Case Presentation}

\section{Case 1}

A 63-year-old man was admitted to our hospital with first ever complaint of back pain in February 2015. He had a medical history of surgery for esophageal cancer without recurrence. He had a smoking history of 40 cigarettes per day for 40 years and an al- cohol history of 8 cups of whisky per day for 35 years. Abdominal ultrasonography (US) showed dilation of the MPD. Contrast-enhanced computed tomography (CE-CT) and magnetic resonance cholangiopancreatography (MRCP) showed localized stenosis of the MPD in the pancreatic body with distal dilation but no obvious tumor at the site of stenosis (Fig. 1a). Endoscopic US (EUS) showed no mass lesion around the stenosis (Fig. 1b). Endoscopic retrograde pancreatography (ERP) was attempted. However, deep cannulation was unsuccessful, and pathological diagnosis was not performed. Although these findings suggested high-grade PanIN or small pancreatic cancer, the patient was followed up, as there was no definitive diagnosis.

Follow-up examinations were performed for 15 months (3month intervals) using CT and MRCP. There were no obvious changes in the form of the MPD at follow-up (Fig. 1c). Similar findings were observed by EUS (Fig. 1d). However, ERP was reperformed because of the potential for high-grade PanIN. ERP showed no evidence of abnormalities in the pancreatic head but localized irregular stenosis in the pancreatic body with distal MPD dilation (Fig. 1e). Cytology of pancreatic juice was obtained by aspiration, brushing, and through endoscopic nasopancreatic drainage (ENPD), although only the brush cytology sample showed adenocarcinoma.

The patient underwent a distal pancreatectomy with splenectomy along the line of the right side of the portal vein. Pancreatography of the resected specimen showed irregular stenosis around the margin and dilation of the distal MPD and branches (Fig. 1f). The branches around the MPD stenosis had poor contrast. Microscopically, postoperative pathology indicated diffuse lesions corresponding to high-grade PanINs in the irregular MPD stenosis and surrounding branches (Fig. 1g, h). Immunohistochemical study indicated positive MUC-5AC and -6 staining and negative MUC-1 and - 2 staining. Severe fibrosis with numerous inflammatory cells and disappearance of acinar cells were observed around the MPD stenosis. An 8-mm diameter branch-type intraductal papillary mucinous adenoma (IPMA) was observed at the tail of the pancreas but separate from the high-grade PanIN of the MPD. Final diagnosis was high-grade PanIN of the main and branch MPD (Tis N0 M0, stage 0 [UICC-7]) and IPMA. The postoperative course was uneventful, and the patient was discharged and is doing well without recurrence at 10 months following surgery.

Case 2

In a 77-year-old man, first ever symptoms of MPD dilation were detected in October 2012, 25 months before referral to our hospital. He had a medical history of cerebral infarction and atrial fibrillation. He had a smoking history of 40 cigarettes per day for 55 years and an alcohol history of 2 bottles of beer per day for 50
Fig. 1. Case 1. a Magnetic resonance cholangiopancreatography (MRCP) demonstrated stenosis of the main pancreatic duct (MPD) in the pancreatic head and body and distal MPD dilation. b Endoscopic ultrasonography (EUS) revealed no evidence of a mass lesion around the MPD stenosis. c MRCP at 15-month follow-up showed no change in MPD stenosis and distal dilation (see a). d EUS at 15-month follow-up showed no change in MPD form. e Endoscopic retrograde pancreatography demonstrated no apparent abnormalities in the pancreatic head of the MPD but local- ized irregular stenosis in the pancreatic body. $\mathbf{f}$ Postoperative pancreatography revealed irregular stenosis around the margin and dilation of the distal MPD and branches. The branches around the MPD stenosis had poor contrast. g, h Macroscopic pathological findings in the MPD, with evidence of high-grade pancreatic intraepithelial neoplasia. The irregular stenosis of the MPD in the pancreatic body was associated with severe fibrosis and numerous inflammatory cells.

(For figure see next page.)
82

Oncology 2017;93(suppl 1):81-86 DOI: $10.1159 / 000481237$
Yamao et al. 

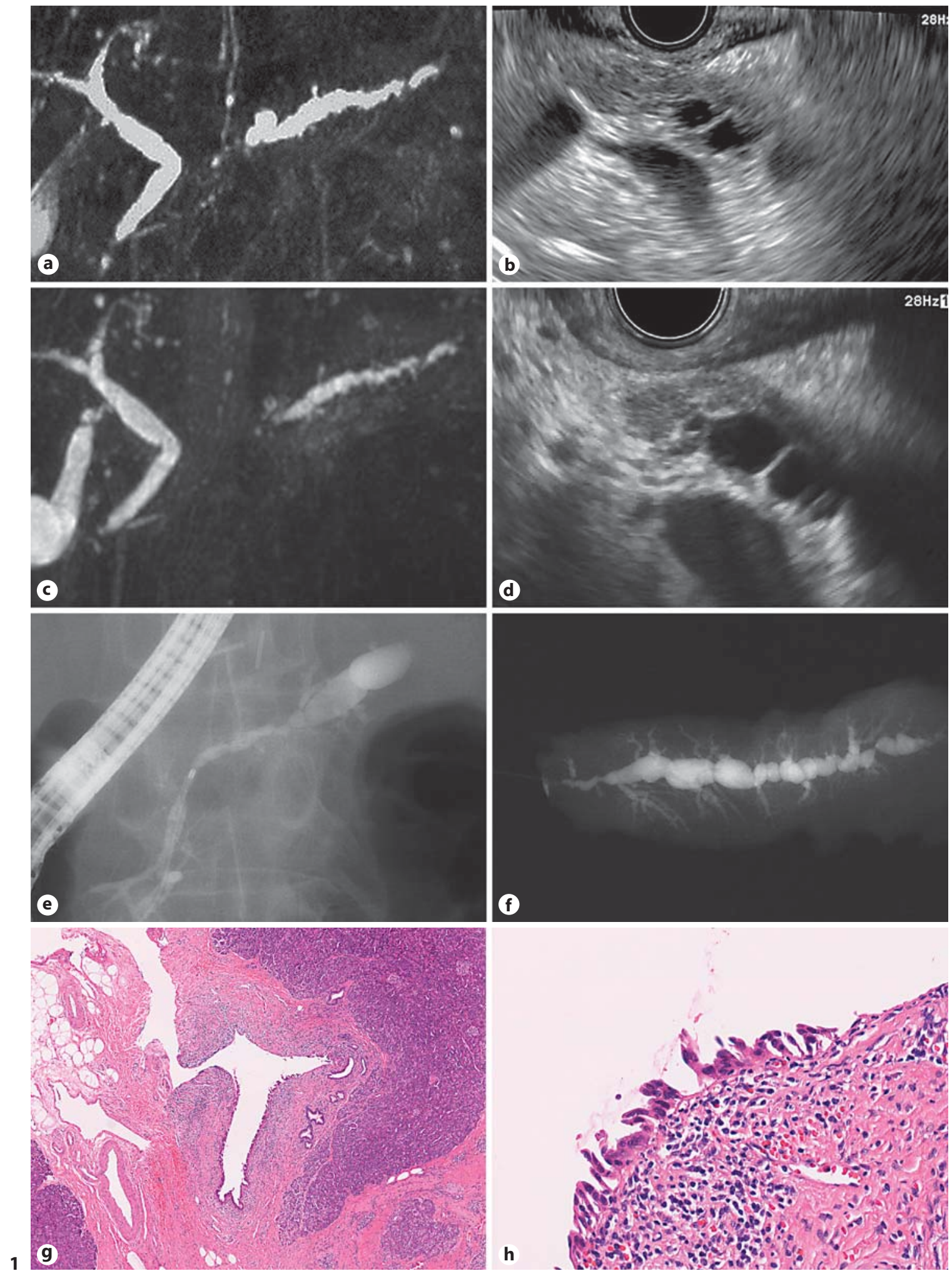

High-Grade PanIN without

Morphological Changes of the MPD
Oncology 2017;93(suppl 1):81-86 DOI: $10.1159 / 000481237$ 

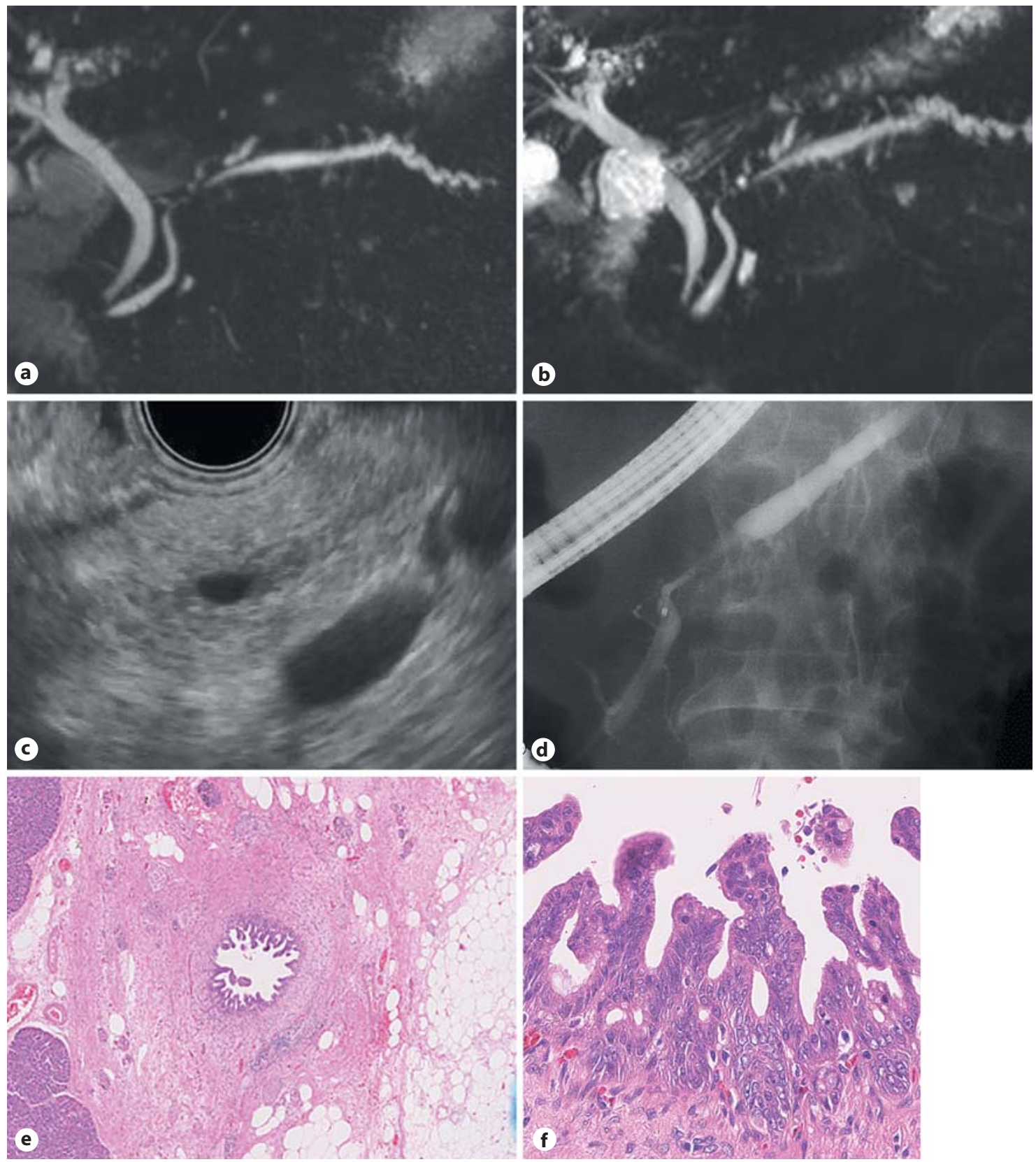

Fig. 2. Case 2. a Magnetic resonance cholangiopancreatography (MRCP) revealed MPD stenosis in the pancreatic head and distal MPD dilation. $\mathbf{b}$ MRCP at 25-month follow-up showed no change of the MPD stenosis and slight worsening of the distal dilation. $\mathbf{c}$ EUS demonstrating the MPD stenosis without a mass lesion. $\mathbf{d}$ ERP demonstrating the localized irregular stenosis of the MPD in the pancreatic body. e, $\mathbf{f}$ Macroscopic pathological findings of the branch pancreatic duct, with evidence of high-grade pancreatic intraepithelial neoplasia.

years. He had received several examinations in October 2012 by a local doctor, with abdominal US showing a 5-mm MPD dilation. CE-CT and MRCP showed localized stenosis of the MPD in the pancreatic head and dilation with distal MPD, although no obvious tumor was observed (Fig. 2a). The diagnosis at that time was MPD stenosis caused by chronic pancreatitis, and the patient was recommended for observation. Ultrasonography was performed 1 year after, with no change in the form of the MPD. However, the patient was referred to our hospital because of a mild change in the size of the MPD in November 2014 (25 months after his initial visit). CE-CT and MRCP showed stenosis of the MPD in the pancreatic head and distal MPD dilation, although no obvious tumor 
at the site of stenosis was observed and the form of the MPD was similar to that 25 months before (Fig. 2b). EUS showed no mass lesion around the MPD stenosis (Fig. 2c). Nevertheless, we performed ERP given the potential for a high-grade PanIN. ERP showed localized irregular stenosis of the MPD in the pancreatic body (Fig. 2d). Aspiration cytology and ENPD repeated cytology were performed, with 1 aspiration cytology sample showing suspected adenocarcinoma. The patient underwent a subtotal stomach-preserving pancreatoduodenectomy.

Microscopically, postoperative pathology indicated a localized lesion with high-grade PanIN in the branch duct around the MPD stenosis (Fig. 2e, f). Immunohistochemical study indicated positive MUC- 6 staining and negative MUC-1, -2 , and -5AC staining. Severe fibrosis with numerous inflammatory cells and disappearance of acinar cells were observed around the MPD stenosis. A 10mm diameter branch-type IPMA was observed at the head of the pancreas, separate from the high-grade PanIN of the MPD. The final diagnosis was high-grade PanIN of the branch duct (Tis N0 M0, stage 0 [UICC-7]) and IPMA. The postoperative course was uneventful, and the patient was discharged and is doing well without recurrence at 32 months following surgery.

\section{Discussion}

Patients with pancreatic cancer have an extremely poor prognosis because of the difficulty of early detection and the highly progressive tumor speed. Nevertheless, the 5 -year survival rate of patients with high-grade PanIN is $85.8 \%$ [1]. Thus, early detection of pancreatic cancer is critical for improved prognosis. PanINs are defined as non-mass-forming lesions found only in the pancreatic ductal epithelium [2]. Detection of high-grade PanIN involves the presence of an abnormal MPD form, including localized MPD stenosis with distal MPD dilation, focal ductal branch dilation, and cyst formation. Exacerbation of these findings with tumor progression is also a sign of high-grade PanIN.

However, there was no evidence of new tumor appearance or exacerbation of the MPD form at long-term followup in our 2 cases of high-grade PanIN (case 1, 15 months; case 2, 25 months), indicating no or slight progression during this period. The long follow-up period in case 1 was because there was no definitive diagnosis of pancreatic cancer (ERP was unsuccessful), while case 2 was followed up in a nonspecialized hospital where the initial MPD abnormality was detected. Although there was no definitive diagnosis of PanINs at the initial hospital visits, if present, these findings without morphological changes of the MPD suggest that the progression time of PanINs was very slow.

In a genomic analysis of autopsy patients with pancreatic cancer to evaluate the clonal relationships between primary and metastatic cancers, Yachida et al. [4] report-

High-Grade PanIN without

Morphological Changes of the MPD ed a progression time between tumor genesis and PanIN (until the progression of the invasive carcinoma) of 11.7 years. These data support our findings of the presence of high-grade PanINs during the 1 - to 2 -year period from initial findings to surgery in our 2 cases. To our knowledge, there is only 1 reported case of high-grade PanIN in which diagnosis of malignancy was difficult and with a follow-up of more than 1 year. However, in that case, the form of the MPD changed markedly at 2.5 years after initial examination [5]. Therefore, our 2 cases without morphological changes of the MPD are important for clinical practice.

As high-grade PanINs are non-mass-forming lesions and are difficult to visualize, EUS-guided fine-needle aspiration biopsy, which is generally performed to diagnose pancreatic cancer, is not effective. There are previous reports using cytology with pancreatic juice, including repeated cytology of pancreatic juice obtained using an ENPD tube and brush cytology, for diagnosis of highgrade PanINs and small pancreatic cancer. Using repeated cytology with an ENPD tube, Iiboshi et al. [6] reported a sensitivity, specificity, and accuracy of detecting highgrade PanIN and small pancreatic cancer of 100, 83.3, and 95\%, respectively, while Mikata et al. [7] reported a sensitivity, specificity, and accuracy of detecting pancreatic cancer, including advanced pancreatic cancer, of 80, 100, and $87 \%$, respectively, which was significantly higher than the sensitivity, specificity, and accuracy obtained when using single cytology. Further, 1 study reported diagnosis using brush cytology in a high-grade PanIN patient [5]. Thus, these methods can be used to directly and efficiently collect the specimens of pancreatic duct epithelium lesions, which are otherwise difficult to perform using EUS-guided fine-needle aspiration.

The 2016 revised clinical guidelines for pancreatic cancer using evidence-based medicine published by the Japan Pancreas Society suggest a number of risk factors for pancreatic cancer, including family history of pancreatic cancer, diabetes, obesity, chronic pancreatitis, intraductal papillary mucinous neoplasm, pancreatic cysts, smoking, and heavy drinking [8]. Our 2 cases had intraductal papillary mucinous neoplasm, smoking, and heavy drinking as risk factors. Thus, cases with localized MPD stenosis and distal MPD dilation should be closely monitored for potential high-grade PanIN, even if the form of the MPD does not change at follow-up observation. Further, patients with some risk factors for pancreatic cancer should be more carefully monitored. In such cases, ERP with pancreatic juice cytology should be considered for definitive diagnosis.

Oncology 2017;93(suppl 1):81-86 DOI: $10.1159 / 000481237$ 
In conclusion, we reported 2 cases of high-grade PanIN without morphological changes of the MPD over relatively long periods. When findings of localized MPD stenosis and distal MPD dilation are detected, a definite diagnosis of high-grade PanINs should be considered by pancreatic juice cytology. Even if a definite diagnosis cannot be obtained, strict follow-up observational study should be performed. Re-examination should also be considered, particularly in cases with some risk factors for pancreatic cancer, even if there is no change in the MPD form.

\section{Statement of Ethics}

Written informed consent was obtained from both patients for publication of this case report.

\section{Disclosure Statement}

The authors declare that they have no competing interests.

\section{Funding Sources}

No funding was received.

\section{Author Contributions}

K.Y. drafted the manuscript. A.N., S.O., K.K., K.M., and T.M. performed clinical data acquisition. H.I. contributed to the treatment of the patient. M.T., I.M., Y.T., and M.K. critically revised the manuscript for important intellectual content. T.C. performed the pathological analysis. All authors read and approved the final manuscript.

\section{References}

1 Egawa S, Toma $\mathrm{H}$, Ohigashi $\mathrm{H}$, Okusaka T, Nakao A, Hatori T, et al: Japan Pancreatic Cancer Registry; 30th year anniversary: Japan Pancreas Society. Pancreas 2012;41:985-992.

2 Hruban RH, Adsay NV, Albores-Saavedra J, Compton C, Garrett ES, Goodman SN, et al: Pancreatic intraepithelial neoplasia: a new nomenclature and classification system for pancreatic duct lesions. Am J Surg Pathol 2001;25:579-586.

3 Basturk O, Hong SM, Wood LD, Adsay NV, Albores-Saavedra J, Biankin AV, et al: A revised classification system and recommendations from the Baltimore Consensus Meeting for Neoplastic Precursor Lesions in the Pancreas. Am J Surg Pathol 2015;39:1730-1741.
4 Yachida S, Jones S, Bozic I, Antal T, Leary R, Fu B, et al: Distant metastasis occurs late during the genetic evolution of pancreatic cancer. Nature 2010;467:1114-1117.

5 Seki M, Ninomiya E, Hayashi K, Gotoh H, Koga R, Saiura A, et al: Widespread and multifocal carcinomas in situ (CISs) through almost the entire pancreas: report of a case with preoperative cytological diagnosis. Langenbecks Arch Surg 2010;395:589-592.
6 Iiboshi T, Hanada K, Fukuda T, Yonehara S, Sasaki T, Chayama K: Value of cytodiagnosis using endoscopic nasopancreatic drainage for early diagnosis of pancreatic cancer: establishing a new method for the early detection of pancreatic carcinoma in situ. Pancreas 2012;41:523-529.

7 Mikata R, Ishihara T, Tada M, Tawada K, Saito M, Kurosawa J, et al: Clinical usefulness of repeated pancreatic juice cytology via endoscopic naso-pancreatic drainage tube in patients with pancreatic cancer. J Gastroenterol 2013;48:866-873.

8 Yamaguchi K, Okusaka T, Shimizu K, et al: Clinical practice guidelines for pancreatic cancer 2016 from the Japan Pancreas Society: a synopsis. Pancreas 2017;46:595-604. 